\title{
Proprotein Convertase Subtilisin/Kexin Type 9 Measurement
}

National Cancer Institute

\section{Source}

National Cancer Institute. Proprotein Convertase Subtilisin/Kexin Type 9 Measurement.

NCI Thesaurus. Code C120647.

The determination of the proprotein convertase subtilisin/kexin type 9 present in a sample. 